\title{
Metal-Catalyzed Oxidation and Photo-oxidation of Glucagon
}

\author{
Jian Zhang ${ }^{1,2}$
}

Received 4 August 2015; accepted 21 September 2015; published online 5 October 2015

\begin{abstract}
The oxidation of glucagon by the $\mathrm{H}_{2} \mathrm{O}_{2} / \mathrm{Cu}^{2+}$ system and by simulated sunlight was studied using HPLC-MS methodologies. It was found that copper ion-catalyzed oxidation is much faster in the residue 1-12 region than in photo-oxidation, but it is slower than photo-oxidation in the residue 18-29 region. This difference is due to the unique feature of the primary sequence of glucagon. The residue 1-12 region contains His- 1 and Asp- 9 that can bind to $\mathrm{Cu}^{2+}$ ions and catalyze the oxidation of His-1 and Tyr-10, while the residue 18-29 region lacks these charged residues near the liable Met-27 and Trp-25 and hence no catalysis by the neighboring groups occurs. Fragment (residue 13-17) was more stable than the other regions of the peptide toward photo-oxidation because it contains only one oxidizable residue, Tyr- 13 . These findings may help explain the mechanism of action of glucagon and provide some hints for the development of effective anti-diabetic drug molecules and stable glucagon formulations.
\end{abstract}

KEY WORDS: copper ion-catalyzed oxidation; drug stability; glucagon; molecular mechanism of action; photo-oxidation.

\section{INTRODUCTION}

Glucagon is a 29 -amino acid hormone produced by the pancreatic cells in response to low blood glucose. It is used as an effective drug to treat hypoglycemia (1). Because the raw material of glucagon (not for medical use) oxidizes readily, its suppliers advise that glucagon be stored at freezing temperature avoiding light exposure. Medical use Glucagon Kit is manufactured as a lyophilized formulation with lactose which can be stored at room temperature for a specified time away from light and moisture. After the expiration day, degradation of the product will occur. In addition, oxidation degradation can occur during the manufacture process and after reconstitution of the product prior to dosing. On the other hand, a small amount of metal ions such as iron and copper may be present as impurities in the excipients in contact with the drug or among the container leachables. These metal ions are known to catalyze the oxidation reaction of protein pharmaceuticals. Glucagon itself may also carry some metal ions if it is not purified thoroughly. To date, there is little published information on glucagon oxidation although private industrial data may exist. Therefore, a study to understand the oxidation mechanisms will be beneficial to pharmaceutical scientists in order to develop the stable glucagon drug product. This research briefly studies the copper ion-catalyzed and photooxidation of glucagon. Copper is selected in the study as opposed to iron due to the limited amount of glucagon available that allows the study of only one metal ion; the effect of

\footnotetext{
${ }^{1}$ Dr. J. Consulting, Atlanta, Georgia 30341, USA.

${ }^{2}$ To whom correspondence should be addressed. (e-mail: jzhang_mail04@yahoo.com)
}

iron is expected to be similar to copper but with weaker catalytic power.

Biologists reported previously that the binding of glucagon to its receptors on the pancreatic and hepatic cells triggers a series of biochemical events such as cAMP stimulation, membrane depolarization, and calcium channel opening of the cells (2-5), which can lead to type II diabetes. However, the molecular mechanism of these phenomena is unclear. Therefore, this research work also tries to provide some evidence if the metal ion-catalyzed oxidation of glucagon is related to the biological function of this hormone because both iron and copper ions are in the body and in the cell culture fluids used in the cellular studies cited in the references.

\section{MATERIALS AND METHODS}

\section{Materials}

Porcine glucagon, cupric sulfate, and $29 \%$ ammonia solution were purchased from Sigma Chemical Company, St. Louis, MO. $30 \%$ hydrogen peroxide was purchased from Aldrich Chemicals, Milwaukee, WI. HPLC-grade trifluoroacetic acid (TFA) was obtained from J.T. Baker Chemicals, Phillipsburg, NJ. HPLC-grade ammonia acetate was from Fisher Chemicals, Fair Lawn, NJ. Sequence-grade trypsin was purchased from Promega, Madison, WI.

\section{Metal-Catalyzed Oxidation of Glucagon}

Glucagon was dissolved in $0.01 \mathrm{M}$ ammonia acetate $(\mathrm{pH}$ 6.8) at a concentration of $0.51 \mathrm{mg} / \mathrm{ml}$. $\mathrm{CuSO}_{4}$ and $\mathrm{H}_{2} \mathrm{O}_{2}$ were added into the glucagon solution to obtain a concentration of $25 \mu \mathrm{M}$ and $0.15 \%$, respectively. The oxidation reaction was 
allowed to proceed at $25 \pm 0.5^{\circ} \mathrm{C}$ for $4 \mathrm{~h}$. The reaction was stopped by adding $1.5 \mathrm{mM}$ butylated hydroxyanisole, and the final concentration of glucagon was $0.17 \mathrm{mg} / \mathrm{ml}$. The oxidized glucagon was digested by trypsin at $37^{\circ} \mathrm{C}$. The $\mathrm{pH}$ of the digest mixture was adjusted to 7.8, and the ratio of glucagon to trypsin was $25: 1(w / w)$. After $16 \mathrm{~h}$ of incubation, the tryptic digest of glucagon was analyzed by liquid chromatography/ mass spectrometry (LC/MS).

\section{Photo-oxidation of Glucagon}

Glucagon was dissolved in $0.01 \mathrm{M}$ ammonia acetate at a concentration of $0.34 \mathrm{mg} / \mathrm{ml}$. The $\mathrm{pH}$ of the sample solutions was adjusted to 9.0 by the addition of ammonia hydroxide, and the concentration of glucagon was diluted to $0.17 \mathrm{mg} / \mathrm{ml}$ after the $\mathrm{pH}$ adjustment. The solution was then exposed to simulated solar radiation $(290-800 \mathrm{~nm})$ in a Heraeus Xenon Lamp Suntester (Phoenix, AZ), with the irradiance set at $760 \mathrm{~W} / \mathrm{m}^{2}$. The temperature inside the Suntester was maintained at $30 \pm 1^{\circ} \mathrm{C}$. The oxidized glucagon solution was removed from the Suntester after $4 \mathrm{~h}$ and digested by trypsin at $37^{\circ} \mathrm{C}$. The $\mathrm{pH}$ of the digest mixture was adjusted to 7.8 , and the ratio of glucagon to trypsin was 25:1 (w/w). After $16 \mathrm{~h}$ of incubation, the tryptic digest of glucagon was analyzed by LC/MS.

\section{LC/MS Analysis of the Tryptic Digest of Glucagon}

The analytical method employed a Hewlett Packard 1100 LC system coupled with a diode array detector and an atmospheric pressure-electrospray (API-ES) mass spectrometric detector. The tryptic digest of glucagon was eluted on an YMC-AQ reversed- phase column $(5 \mu, 100 \AA, 4.5 \times 250 \mathrm{~mm})$. A linear gradient was performed starting with an initial mobile phase composition of $0.01 \% \mathrm{TFA} /$ methanol $(90: 10)$ to a final composition of $0.01 \% \mathrm{TFA} / \mathrm{methanol}(20: 80)$ over $50 \mathrm{~min}$. The flow rate was $0.5 \mathrm{ml} / \mathrm{min}$. API-ES mass spectra of the digest of glucagon were acquired in the $\mathrm{m} / \mathrm{z}$ range from 100 to 2000 following chromatographic separation. The fragmentation voltage of the mass spectrometer was adjusted between 70 and $180 \mathrm{~V}$ to optimize the abundance of the fragment ions. The spray chamber temperature was $350^{\circ} \mathrm{C}$, the drying gas flow was $12.0 \mathrm{l} / \mathrm{min}$, and the nebulization pressure was $50 \mathrm{psi}$.

\section{RESULTS}

\section{Chromatography of the Tryptic Digest of Unoxidized Glucagon}

The tryptic digest of glucagon has been characterized previously by a different chromatographic method (6). As shown by the arrows pointing to the sequence of glucagon in Scheme 1, there are three sites for cleavage by trypsin resulting in four fragments and an arginine residue. All four tryptic fragments were eluted and identified by their masses using the new LC/MS method used in this study, and they were the same as the ones shown in the reference. The chromatogram of the digest fragments of non-oxidized glucagon is illustrated in Fig. 1. The other unwanted chromatographic peaks in Fig. 1 belonged to trypsin. Trypsin split into multiple peaks in mass spectrometry due to its large molecular weight

\section{Glucagon (MW 3482):}

$$
\begin{aligned}
& \text { His-Ser-Gln-Gly-Thr-Phe-Thr-Ser-Asp-Tyr-Ser-Lys-Tyr Leu-Asp- } \\
& \text { Ser-Arg-Arg-Ala-Gln-Asp-Phe-Val-Gln-Trp-Leu-Met-Asn-Thr } \\
& \text { Tryptic Digestion }\left(\mathrm{pH} 7.8,37^{\circ} \mathrm{C}\right) \\
& \text { His-Ser-Gln-Gly-Thr-Phe-Thr-Ser-Asp-Tyr-Ser-Lys (MW 1357) } \\
& + \\
& \text { Tyr Leu-Asp-Ser-Arg (MW 653) } \\
& + \\
& \text { Arg-Ala-Gln-Asp-Phe-Val-Gln-Trp-Leu-Met-Asn-Thr (MW 1352) } \\
& + \\
& \text { Arg-Arg-Ala-Gln-Asp-Phe-Val-Gln-Trp-Leu-Met-Asn-Thr (MW 1510) } \\
& + \\
& \text { Arg (MW 175) }
\end{aligned}
$$

Scheme 1. Sequence of glucagon with arrows pointing to the position of cleavage by trypsin

and multiple charges. These peaks differed from the glucagon peaks in that their intensity did not change before and after oxidation.

\section{Chromatography of Oxidized Glucagon}

When glucagon was oxidized by the $\mathrm{H}_{2} \mathrm{O}_{2} / \mathrm{Cu}^{2+}$ system at pH 6.8, Fragment (residue 1-12) was completely destroyed as shown in Fig. 2a. Figure 3 illustrates the percent degradation of glucagon which was calculated from the decrease of peak intensity from the LC/MS chromatograms of the tryptic digests. The degradation of the residue 1-12 region can be attributed to the oxidation of His-1 and Tyr-10 that is adjacent to the negatively charged Asp-9 residue which may bind to the $\mathrm{Cu}^{2+}$ ions. The degradation of histidine by the $\mathrm{H}_{2} \mathrm{O}_{2} / \mathrm{Cu}^{2+}$ system and the binding of the carboxyl group to $\mathrm{Cu}^{2+}$ ion that catalyzes oxidation of nearby tyrosine (in Glu-Tyr and $\mathrm{N}$ acetyltyrosine) have been studied and reported previously $(7,8)$. On the contrary, when glucagon was oxidized by simulated sunlight at $\mathrm{pH} 9$, Fragment (residue 1-12) became less susceptible to oxidation than in metal-catalyzed degradation (Figs. 2b and 3). Fragment (residue 13-17) was slightly more rapidly oxidized in metal-catalyzed degradation than in photooxidation (Fig. 3) possibly because of the binding of $\mathrm{Cu}^{2+}$ ion by Asp-15 that catalyzed the oxidation of Tyr-13. The chromatographic peaks of Fragment (residue 18-29) and Fragment (residue 17-29) decreased upon oxidation due to the destruction of Met-27 and Trp-25 residues. However, the rate of metal-catalyzed degradation in this region was lower than that of photo-oxidation because there is no charged group near the liable Met-27 and Trp-25 residues that can bind to $\mathrm{Cu}^{2+}$. Asp-21 and the C-terminus can potentially interact with the copper ion, but it is not next to an oxidizable residue which would cause significant degradation. Fragment (residue 13-17) was more stable than the other fragments toward photo-oxidation because it contains only one oxidizable residue Tyr-13 which degraded to a lesser degree than His-1, Met-27, and Trp-25. The degradant detected in metalcatalyzed oxidation has an $\mathrm{m} / \mathrm{z}$ value of 1406 , and the degradant observed in photo-oxidation has an $\mathrm{m} / \mathrm{z}$ value of 1415. However, the exact locations of the chemical modification are not immediately clear. 
MSD1 TIC, MS File (08_17_02\JZ000006.D) API-ES, Pos, Scan, Frag: 180

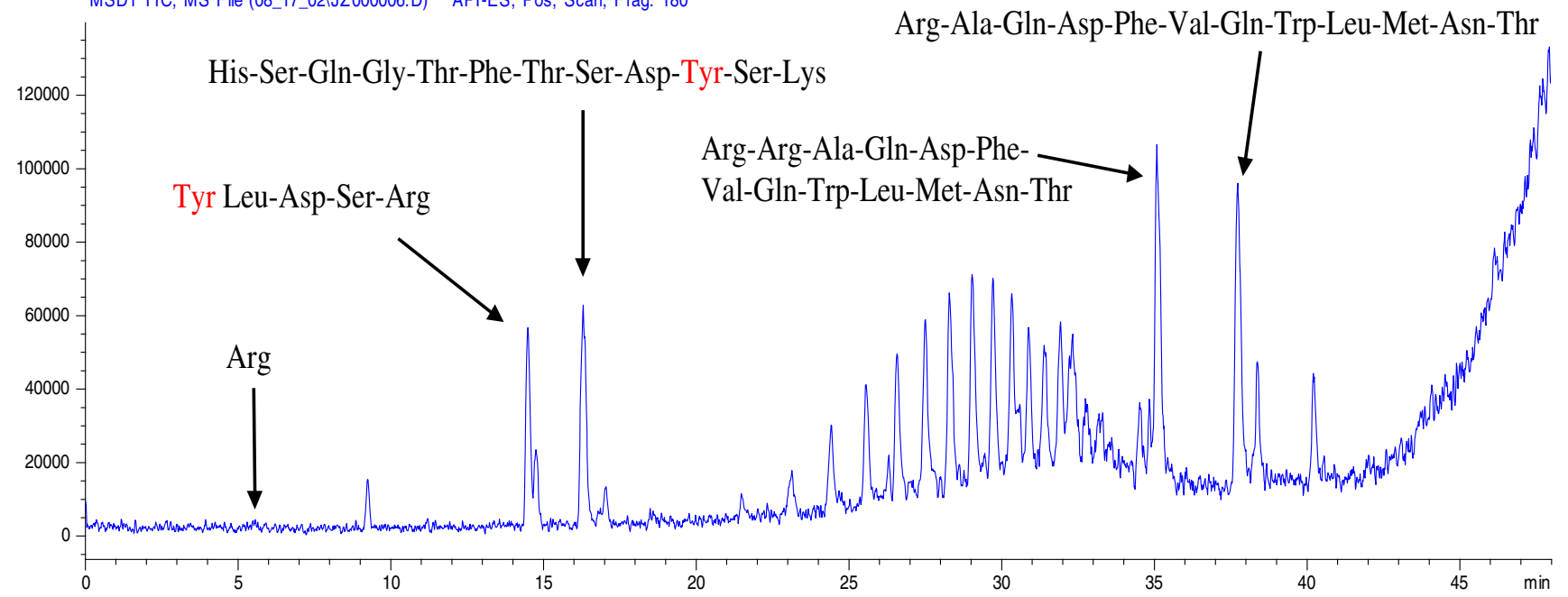

Fig. 1. HPLC chromatograms of the tryptic digest of unoxidized glucagon $(0.34 \mathrm{mg} / \mathrm{ml})$ with MS detection

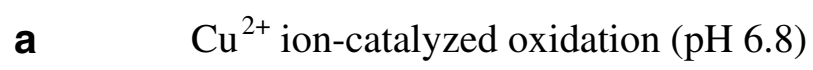

MSD1 TIC, MS File (08_22_021JZ000002.D) API-ES, Pos, Scan, Frag: 180

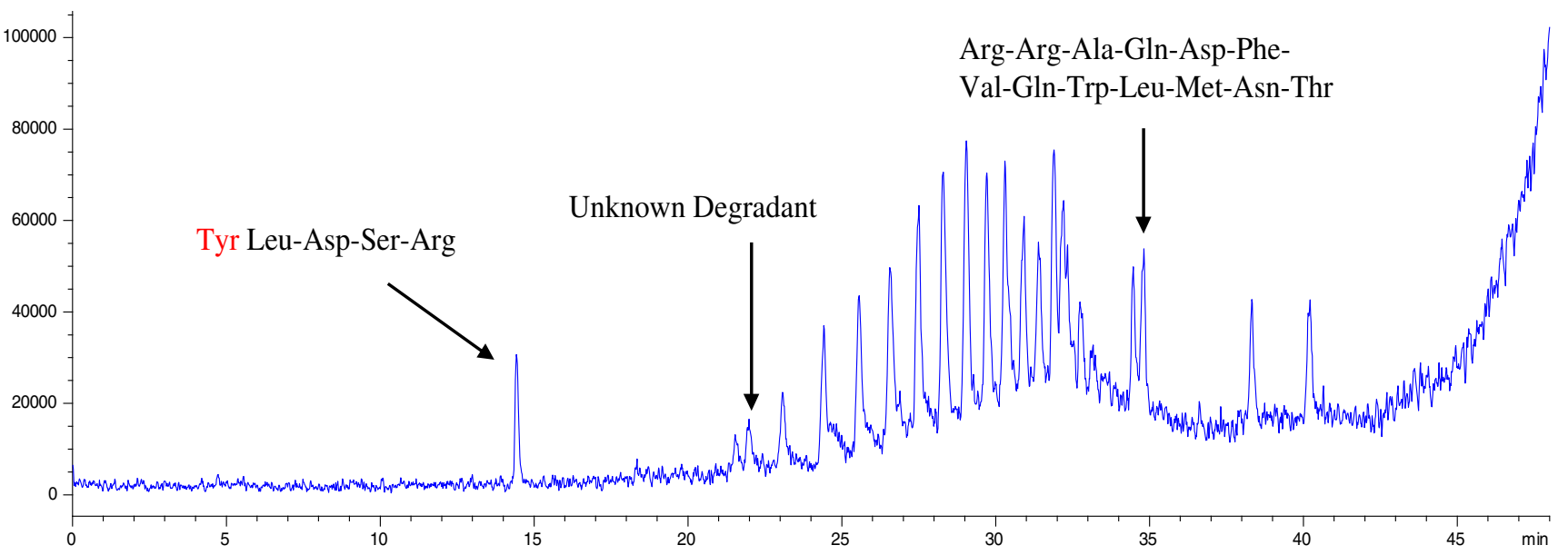

b Photo-oxidation ( $\mathrm{pH} 9.0)$

MSD1 TIC, MS File (08_20_02IJZ000002.D) API-ES, Pos, Scan, Frag: 180

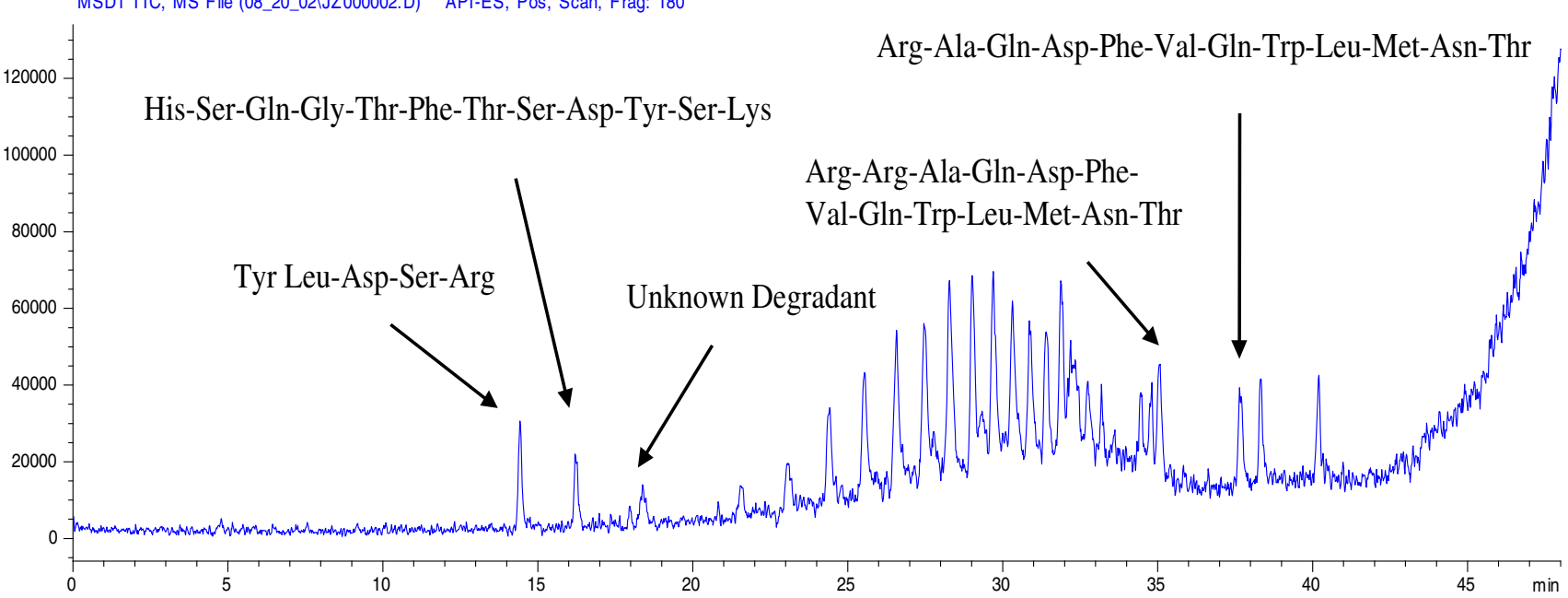

Fig. 2. HPLC chromatograms of the tryptic digest of oxidized glucagon. a $\mathrm{Cu}^{2+}$ ion-catalyzed oxidation ( $\mathrm{pH}$ 6.8). b Photo-oxidation (pH 9.0) 


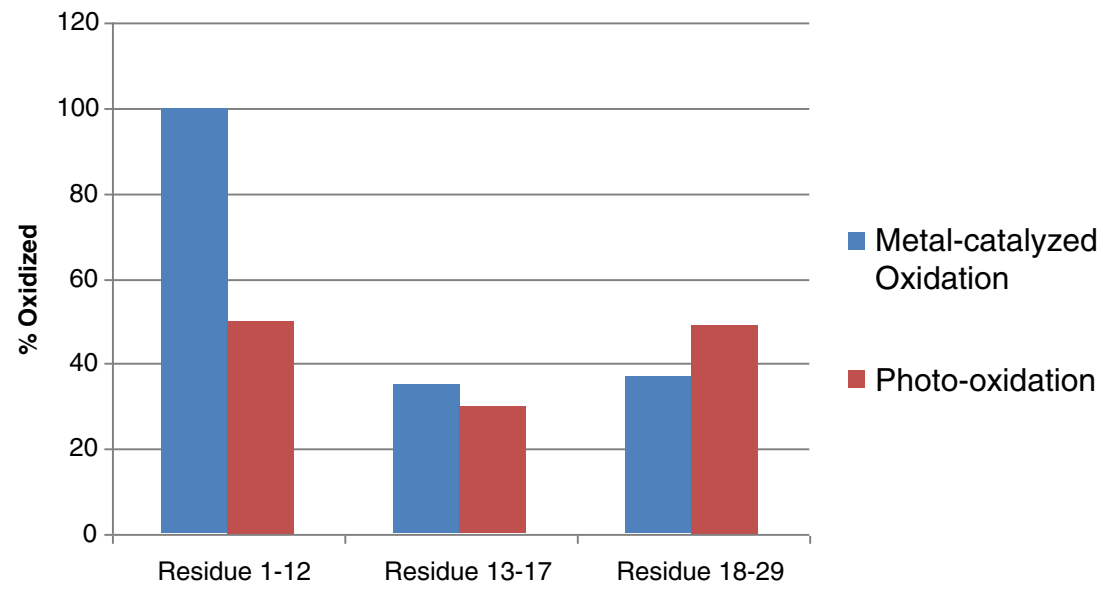

Fig. 3. Percentage of glucagon remaining after oxidation for $4 \mathrm{~h}$

\section{DISCUSSION}

ATP synthesis involves the transfer of electrons from outside of the cell to the matrix and the movement of protons in the opposite direction through the cell membrane (9-11). The observation of the copper ion-catalyzed oxidation of glucagon shown in this study probably explains the cellular biochemical events mentioned earlier because the free radicals and proton concentration change generated by this reaction can have an impact on cellular ATP/cAMP synthesis and metabolism. Indirect evidence was presented in earlier studies that His-1 and Asp-9 were critical for the glucagon function and removal of these two residues would almost inactivate the hormone $(5,12,13)$. This is probably because His-1 and Asp-9 of glucagon bind to metal ions such as $\mathrm{Cu}^{2+}$ and catalyze free-radical oxidation reactions. Recent studies using computer modeling demonstrated that the middle to C-terminus region of glucagon was more important for receptor binding while the $\mathrm{N}$-terminus is responsible for signal transduction and triggering of structural changes of the receptor (14-16). The finding of this oxidation experiment is consistent with the results of these studies, i.e., the region of residue $1-12$ is more prone to metal-catalyzed oxidation than the region of residue 18-29. The correlation between the copper catalysis near the N-terminus (e.g., His-1) and the activity of glucagons will need to be validated in cellular studies in the future. Nonetheless, the current study serves as a bridging study leading to this future work.

Furthermore, it is hypothesized that the cellular biochemical reactions discussed above may be inhibited by drugs that can stop the metal-catalyzed oxidation reaction of glucagon. A recent study shows that the anti-diabetic drug metformin is efficacious because it can complex with the copper ion in the liver cell to reduce AMP-activated protein kinase (AMPK)dependent signaling, glucose production, and mitochondrial respiration (17). It will be interesting to find out if metformin can slow the copper ion-catalyzed oxidation of glucagon as a potential mechanism of action of this drug.

\section{CONCLUSION}

Glucagon can be oxidized by the $\mathrm{H}_{2} \mathrm{O}_{2} / \mathrm{Cu}^{2+}$ system and simulated sunlight. The rate of copper ion-catalyzed oxidation is much higher in the residue $1-12$ region than in photo- oxidation, but it is lower than the rate of photo-oxidation in the residue 18-29 region. This difference is due to the unique feature of the primary sequence of glucagon. The residue 1-12 region contains histidine and aspartic acid that can bind to $\mathrm{Cu}^{2+}$ ion and catalyze the oxidation of His-1 and Tyr-10, while the residue 18-29 region lacks these charged residues near the liable Met-27 and Trp-25 and hence no catalysis by the neighboring groups occurs. Fragment (residue 13-17) was more stable than the other regions of the peptide toward photooxidation because it contains only one oxidizable residue, Tyr13. These findings may help explain the mechanism of action of glucagon and provide some hints on how to develop effective diabetes drugs and stable glucagon formulations.

\section{ACKNOWLEDGMENTS}

The technical and financial supports from Bayer Pharmaceutical Corporation and the University of Connecticut School of Pharmacy are greatly appreciated.

\section{REFERENCES}

1. Information for the user (and the physician), glucagon for injection. Eli Lilly and Company, USA.

2. Sistare FD, Picking RA, Haynes RC. Sensitivity of the response of cytosolic calcium in Quin-2-loaded rat hepatocytes to glucagon, adenine nucleosides, and adenine nucleotides. J Biol Chem. 1985;260(23):12744-7.

3. Ma X et al. Glucagon stimulates exocytosis in mouse and rat pancreatic cells by binding to glucagon receptors. Mol Endocrinol. 2005;19(1):198-212.

4. De Leon DD et al. Exendin-(9-39) corrects fasting hypoglycemia in SUR-1_/_mice by lowering cAMP in pancreatic B-cells and inhibiting insulin secretion. J Biol Chem. 2008;283(38):25786-93.

5. Hinke SA, Pospisilik JA, Demuth HU, Mannhart S, Kuhn-Wache K, Hoffmann T, et al. Dipeptidyl peptidase IV (DPIV/CD26) degradation of glucagon, characterization of glucagon degradation products and DPIV-resistant analogs. J Biol Chem. 2000;275(6):3827-34.

6. Ackermann BL, Coutant JE, Chen TM. Incorporation of tandem mass spectrometric detection to the analysis of peptide mixtures by continuous flow fast atom bombardment mass spectrometry. Biol Mass Spectrom. 1991;20:431-40.

7. Amic A et al. Conversion of amino acid residues in proteins and amino acid homopolymers to carbonyl derivatives by metalcatalyzed oxidation reactions. J Biol Chem. 1989;264(6):3341-6. 
8. Zhang $\mathrm{J}$ and Kalonia DS, The effect of neighboring amino acid residues and solution environments on the oxidative stability of tyrosine in small peptides. AAPS PharmSciTech. 2007; 8(4) Article 102.

9. Biochemistry I: ATP synthesis. CliffsNotes (http:// www.cliffsnotes.com).

10. Mitchell P, Moyle J. Chemiosmotic hypothesis of oxidative phosphorylation. Nature. 1967;213(5072):137-9.

11. Mccarty RE. A plant biochemist's view of $\mathrm{H}^{+}$-ATPases and ATP synthases. J Exp Biol. 1992;172(Pt 1):431-41.

12. Unson CG et al. Roles of aspartic acid 15 and 21 in glucagon action: receptor anchor and surrogates for aspartic acid 9 . Biochemistry. 1994;33(22):6884-7.
13. Unson CG et al. Positively charged residues at positions 12, 17, and 18 of glucagon ensure maximum biological potency. J Biol Chem. 1998;273(17):10308-12.

14. Koth CM et al. Molecular basis for negative regulation of the glucagon receptor. PNAS. 2012;109(36):14393-8.

15. Patterson JT et al. A hydrophobic site on the GLP-1 receptor extracellular domain orients the peptide ligand for signal transduction. Mole Metab. 2013;2:86-91.

16. Parthier $\mathrm{C}$ et al. Passing the baton in class B GPCRs: peptide hormone activation via helix induction? Trends Biochem Sci. 2009;34(6):303-10.

17. Logie L, Harthill J, Patel K, Bacon S, Hamilton DL, Macrae K, et al. Cellular responses to the metal-binding properties of metformin. Diabetes. 2012;61:1423-33. 